\title{
Metabolomic analysis in ophthalmology
}

\author{
Neshe Nazifova-Tasinova ${ }^{a}$, Mladena Radevab ${ }^{b}$ Bistra Galunska ${ }^{a}$, Christina Grupcheva ${ }^{b}$
}

\begin{abstract}
Modern science takes into account phenotype complexity and establishes approaches to track changes on every possible level. Many "omics" studies have been developed over the last decade. Metabolomic analysis enables dynamic measurement of the metabolic response of a living system to a variety of stimuli or genetic modifications. Important targets of metabolomics is biomarker development and translation to the clinic for personalized diagnosis and a greater understanding of disease pathogenesis. The current review highlights the major aspects of metabolomic analysis and its applications for the identification of relevant predictive, diagnostic and prognostic biomarkers for some ocular diseases including dry eye, keratoconus, retinal diseases, macular degeneration, and glaucoma. To date, possible biomarker candidates for dry eye disease are lipid metabolites and androgens, for keratoconus cytokeratins, urea, citrate cycle, and oxidative stress metabolites. Palmitoylcarnitine, sphingolipids, vitamin D related metabolites, and steroid precursors may be used for distinguishing glaucoma patients from healthy controls. Dysregulation of amino acid and carnitine metabolism is critical in the development and progression of diabetic retinopathy. Further work is needed to discover and validate metabolic biomarkers as a powerful tool for understanding the molecular mechanisms of ocular diseases, to provide knowledge on their etiology and pathophysiology and opportunities for personalized clinical intervention at an early stage.
\end{abstract}

Key words: metabolomics, biomarkers, metabolites, ocular diseases

Received: May 22, 2020; Revised: June 19, 2020; Accepted: June 24, 2020; Available online: July 17, 2020

https://doi.org/10.5507/bp.2020.028

(c) 2020 The Authors; https://creativecommons.org/licenses/by/4.0/

${ }^{a}$ Department of Biochemistry, Molecular medicine and Nutrigenomics, Faculty of Pharmacy, Medical University of Varna, 84 Tzar Osvoboditel street, 9000 Varna, Bulgaria

${ }^{b}$ Department of Ophthalmology and Visual Sciences, Faculty of Medicine, Medical University of Varna, 15 Doyran street, 9000 Varna, Bulgaria

Corresponding author: Bistra Tzaneva Galunska, e-mail:bistra.galunska@gmail.com

\section{INTRODUCTION}

Modern science takes into account the phenotype complexity and establishes sets of approaches to track the changes on every possible level. Many "omics" studies have been developed over the last decade. The main ones include the study of the genome of organisms, referred to as "genomics"; the study of the epigenome - "epigenomics", "transcriptomics" includes the whole set of mRNAs present in the system; the proteins in a system represent the "proteomics". Metabolomics studies are focused on the identification and quantification of a set of metabolites in a biological system. Metabolomic analysis enables dynamic measurement of the metabolic response of a living system to a variety of stimuli and genetic modifications. It is widely applied in clinical trials, nutrigenomic studies, functional genomics, and for the assessment of biological effects of different substances.

Modern day metabolomics has been developed over the years starting in the nearly 1960's with analysis of certain molecules using chromatographic techniques. Pauling and Robinson for the first time publish a study in 1970 using gas chromatography analysis of 280 metabolites in urine and 250 in breath. Almost 30 years later, Oliver in 1998 introduced the term "metabolome" in the sci- entific literature. The following year, Nicholson for first time used the term "metabonomics" as a "quantitative measurement of the dynamic multiparametric metabolic response of living systems to pathophysiological stimuli or genetic modifications"'. The term "metabolomics" was introduced after 2000. Currently, there are more than 25000 publications on this topic ${ }^{2}$. In 2005, the first metabolomics database METLIN was established which characterizes the human metabolome and includes all previously and newly discovered metabolites ${ }^{3}$.

In addition to the Human Genome Project, there is a running Human Metabolome Project since 2007 aiming to identify, quantify and catalog all human metabolites.

The current review aims to cover the major aspects of metabolomic analysis and its applications for identification of relevant predictive, diagnostic and prognostic biomarkers for some ocular diseases including dry eye syndrome, keratoconus, retinal diseases, macular degeneration, and glaucoma.

\section{THE GOAL OF METABOLOMICS}

The goal of metabolomics is to conduct a large-scale study on the small molecule metabolites (mass range 50- 
$1500 \mathrm{Da})$, required for the maintenance of a specific cell or tissue function within biofluids and the whole organism. In human studies metabolomic analysis is required to discover reliable and relevant biomarkers for improving diagnosis and prognosis of a disease, to assess efficacy or toxicity of a drug, and to evaluate the metabolic response to endogenous and exogenous factors ${ }^{4,5}$.

\section{CONCEPTUAL APPROACHES}

Two approaches of metabolomic study can be distinguished - untargeted and targeted metabolomics ${ }^{1,6}$.

Untargeted metabolomics compares the metabolome of control and experimental groups to identify differences reflecting specific biological conditions. It aims to detect and analyze in one sample as many metabolites as possible, both known and unknown. The main goal is to classify phenotypes based on metabolite pattern. This approach is related to hypothesis generation and is mainly used for biomarker discovery ${ }^{6}$. As a result, a large amount of highly complex data is generated requiring high performance bioinformatics tools ${ }^{7}$.

The typical untargeted metabolomics workflow includes:

- Profiling for finding metabolites with statistically significant variations within control and test samples. The profiling step usually includes sample preparation, data acquisition, spectral pre-processing, and feature extraction $^{8}$.

- Compound identification using mass-spectrometry databases or spectral libraries for determination the chemical structure of the identified metabolites.

- Interpretation and uncovering biological links between the metabolites using advanced chemometric techniques ${ }^{9}$ and available biological databases ${ }^{7}$.

The other approach, targeted metabolomics is focused on the highly precise qualitative and quantitative target analysis of pre-defined set of specific small molecule metabolites (targets) with known chemical structure and biological role. In the target analysis, the compounds of interest are measured using one specific analytical technique of best performance. In contrast to the untargeted approach, the targeted metabolomics is based on a preestablished hypothesis and is used as a confirmation tool for untargeted studies or for validation of biological pathways $^{10,11}$.

\section{ANALYTICAL PLATFORMS IN METABOLOMICS}

At present there is no single analytical platform or methodology for profiling the entire metabolome. Its complexity including variability of chemical structures and physicochemical characteristics of different metabolites, and the wide dynamic range of physiologic concentrations (from pico- to milimolar) is an analytical challenge for performing metabolomic analysis. In addition, the presence of exogenous metabolites from food, drugs, environ- mental pollutants and the fact that the metabolites have specific tissue or bio-fluid localization, makes metabolomic analysis extremely complicated.

Two major analytical platforms are used in metabolomic studies - nuclear magnetic resonance (NMR) spectroscopy and mass spectrometry (MS) (ref. ${ }^{9}$ ).

\section{Nuclear magnetic resonance spectroscopy}

NMR spectroscopy is based on behavior of atom nuclei spin in strong magnetic field, when they are exctitated by radiofrequency pulse. Since the intramolecular magnetic field around each magnetically inequivalent atom in a molecule cause a difference in the resonance frequency, a details about the electronic structure of a molecule are obtained in a form of different spectral lines. NMR spectroscopy can be used for qualitative and quantitative analysis of molecules and mixtures. With appropriate application of qualitative analysis the molecular structure of unknown compound can be unambiguously determinate. Spectral libraries for comparison with known molecules are also available ${ }^{12}$. The most common NMR technique used in metabolomic analysis is based on measuring of proton $\left({ }^{1} \mathrm{H}\right)$ and carbon $\left({ }^{13} \mathrm{C}\right)$ NMR spectra. By targeting other atoms like phosphorus $\left({ }^{31} \mathrm{P}\right)$ and nitrogen $\left({ }^{15} \mathrm{~N}\right)$, additional information about metabolites in the metabolome can be obtained ${ }^{13,14}$. The peak pattern generated by each metabolite is used for its identification, while the peak areas are used for quantitation of the metabolites in the sample. When 1D-NMR is insufficient due to signal overlapping, a variety of 2D-NMR techniques are available. COSY (COrrelation SpectroscopY) and TOCSY (TOtal Correlation SpectroscopY) are twodimensional NMR methods allowing the identification of neighboring protons in a molecule. Another NMR methods such as $1 \mathrm{H}-1 \mathrm{H}$ NOESY(Nuclear Overhauser Effect SpectroscopY), EXSY (EXchange SpectroscopY) and $1 \mathrm{H}-1 \mathrm{H}$ ROESY (Rotating frame Overhause Effect SpectroscopY) are used for construction of a threedimensional model of a molecule and for detection of conformational changes. The 2D-NMR 13C-13C INADEQUATE (Incredible Natural Abundance Double QUAntum Transfer Experiment), is useful for direct determination of neighboring carbons ${ }^{13,14}$. Due to natural abundance of carbon-13 (1\%) is this experiment very insensitive. Therefore another 2D-technique like HSQC (Heteronuclear Single Quantum Correlation) and HMBC (Heteronuclear Multiple Bond Correlation) are used for determination of carbon Framework connectivity. Advantage of NMR spectroscopy is its ability to provide structural information for the identification of unknown metabolites with little or no sample preparation. A major drawback is its low sensitivity ${ }^{6,13}$.

\section{Mass spectrometry}

Mass spectrometry is an alternative platform to NMRbased metabolomics. It is highly selective and sensitive and is widely used for identification and quantitation of metabolites. MS can typically detect molecules in the femptomolar to attomolar range. Coupled with gas chromatography (GC), ion chromatography (IC) or liquid 
chromatography (LC), MS can routinely analyze numerous compounds in a single sample in one run ${ }^{15}$.

A typical mass spectrometer contains an ion source for the ionization of sample molecules, a mass analyzer to separate all analytes in the sample during full scan analysis, or to filter selected ions based on their mass to charge $(\mathrm{m} / \mathrm{z})$ ratio, and a detector. Usually, ion sources like electrospray ionization (ESI), atmospheric pressure chemical ionization (APCI), and mass analyzers such as time-of-flight (TOF), orbitraps, quadrupoles and ion traps are used in metabolomic analysis. In targeted metabolomic analysis, as powerful analytical platforms for relative quantitation (profiling) and for metabolite identification via MS databases, mostly used are tandem mass spectrometry (MS/MS) and $\mathrm{MS}^{\mathrm{n}}$. These include additional selective generation of ion fragments and their subsequent separation according to individual $\mathrm{m} / \mathrm{z}$ ratios. Using MS/ MS and $\mathrm{MS}^{\mathrm{n}}$ approach, a higher confidence in metabolite identification can be achieved. More advanced MS approaches, like selective reaction monitoring (SRM) enable us to monitor compounds with a certain $\mathrm{m} / \mathrm{z}$ and unique diagnostic ions specific to that compound. This approach allows detection and quantitation of well-characterized compounds in complex matrices such as urine, plasma, blood and tissue samples in metabolomics experiments. A relatively new approach is parallel reaction monitoring (PRM) including high-resolution accurate mass (HRAM) analyzer for detection of all target product ions in parallel. PRM provides high selectivity, sensitivity and throughput quantitation, along with confident targeted confirmation. The method is best suited for quantifying multiple targeted metabolites in complex matrices ${ }^{16}$.

\section{DATA ANALYSIS}

Specialized software is required for the analysis of large amounts of complex data generated by untargeted and targeted metabolomic study. The major aim of metabolomics data analysis is to organize and visualize the data and to construct a hypothesis ${ }^{17}$. The data analysis flow includes statistical analysis, followed by data interpretation and pathway analysis, generation of hypotheses, model construction representing the biological phenomenon, and experimental validation of the hypothesis ${ }^{18}$. Univariate and multivariate statistical analysis is used in the study of metabolomic features and to identify the relationship patterns between them. Supervised and unsupervised pattern-recognition methods are used. The aim of the unsupervised analysis is to find similarity patterns within the data without regard to the sample type. In supervised methods, sample labels are used to identify features or their combinations more tightly associated with the phenotype of interest ${ }^{17,19}$.

The following multivariate methods are most frequently used for statistical analysis of metabolites: principal component analysis (PCA), clustering technique and selforganizing map (SOM), partial least squares regression (PLS), and its extension orthogonal-PLS (OPLS).

Principal component analysis (PCA) is an unsuper- vised technique usually used as a starting point for data analysis. It aims to explain the variance within a big dataset of correlated variables by a smaller number of decorrelated principal components. The result is dimensionality reduction, data visualization, clustering and sample group discrimination. ANOVA-simultaneous component analysis (ASCA) is a combination of ANOVA and PCA. ASCA is used for the analysis of data sets of many simultaneously measured covariates. Cluster analysis is another unsupervised technique for data analysis according to similar patterns and relations. Clustering methods group and visualize data according to intrinsic similarities. Most widely used in metabolomic analysis are k-means clustering and hierarchical clustering ${ }^{19,20}$.

Supervised analysis is usually used for determination of new biomarkers integrating the evidence of multiple metabolites. The aim of the supervised methods is to explain specific metabolite profiles strongly associated with a predefined response. Their usefulness is further evaluated in real clinical settings ${ }^{9}$. Partial least squares (PLS) supervised technique for data analysis is one of the mostly used in metabolomic research. PLS is used for identifying biomarkers and in classifying diseases. Orthogonal partial least square-discriminant analysis (OPLS) is an extension of PLS method. The OPLS method can separate the systematic variation in a variable of interest into two parts: related to the response and unrelated to the response ${ }^{21}$.

A wide variety of software tools are available for the analysis of metabolomic data at the pathway level. Pathway analysis is useful for finding the biological mechanisms among the identified metabolites. Due to the high level of correlation between mRNAs, proteins, and metabolites as well as between the metabolites belonging to a common pathway, correlation-based methods are used to construct metabolite networks based on the relationship patterns in the experiment data ${ }^{22}$.

\section{APPLICATIONS OF METABOLOMICS IN OPHTHALMOLOGY}

Metabolomic profiling provides a practical approach to personalized ophthalmology and has great potential for biomarker discovery for various eye diseases including glaucoma $^{22-24}$, age-related macular degeneration ${ }^{25,26}$, diabetic retinopathy ${ }^{27}$, keratoconus ${ }^{28}$, retinal detachment ${ }^{29}$, uveitis $^{30}$, dry eye ${ }^{31}$, and other ocular diseases. Tear fluid ${ }^{28,32}$, aqueous and vitreous humor ${ }^{33-35}$, cornea ${ }^{36}$, conjunctiva ${ }^{37}$, and lens ${ }^{38}$ samples can be used for metabolomic analysis.

\section{Dry eye syndrome}

Dry eye syndrome (DES) is a multifactorial disease of the ocular surface characterized by a loss of homeostasis of the tear film and accompanied by ocular symptoms such as tear film instability and hyperosmolarity, ocular surface inflammation and damage, neurosensory abnormalities eventually leading to neurodegeneration ${ }^{39}$. Twenty-five percent of patients who visit ophthalmic clinics report symptoms of dry eye, making it an important public health concern. In addition, DES has a significant 
impact on visual function that can negatively affect patients' quality of life and productivity. The disease is multifactorial and has intermittent symptoms ${ }^{40}$. A number of studies have shown several risk factors for the development of DES like an increasing age, gender (especially predominant in females), climate and environmental characteristics. Large numbers of patients with DES also demonstrate signs of meibomian gland dysfunction. Signs and symptoms usually affect both eyes, but might be asymmetric. Patients complain of stinging, burning in the eyes, photophobia, blurred vision, irritation, foreign body sensation, difficulties in wearing contact lenses, watery eyes, eye fatigue and mucoid discharge. If DES is left untreated and becomes severe, it can produce complications that can cause eye damage, resulting in impaired vision or even loss of vision. The diagnosis of DES could be a challenge for many practitioners, especially when the symptoms do not match the signs ${ }^{41}$. The International Dry Eye Workshop has tabulated various approaches for dry eye management, which includes the use of artificial tears, anti-inflammatory agents, tetracyclines, secretogogues, puncta plugs, serum, contact lens, systemic immunosuppressors, and surgical interventions ${ }^{42}$.

A number of methods that determine the quantity and quality of tears, have been used in DES management: slit lamp examination, staining of corneal and conjunctival epithelium with fluorescein, lissamine green, or rose bengal, tear breakup time test, Schrimer test, tear film osmolarity, tear meniscus height, tear protein analysis that measures the lysozyme in the tears, lactoferryn analysis. Recently, abnormally high levels of diadenosine polyphosphate Ap4A I, naturally present in tears, have been found in DES patients ${ }^{43}$. Diadenosine polyphosphates are naturally occurring compounds of the tear, important for the regulation of intraocular pressure and for corneal wound healing. It has been suggested that these substances can be used as biomarkers of DES (ref. ${ }^{43}$ ). ${ }^{1} \mathrm{H}$ NMR-based targeted metabolomic analysis of tear samples of patients with different stage DES and healthy controls before and after 3-month supplementation with antioxidants and omega- 3 essential polyunsaturated fatty acids (EPUFA) revealed significant differences in their metabolite profiles. The main metabolites identified in the tear samples in each group before supplementations were cholesterol, N-acetylglucosamine, glutamate, amino butyrate, choline, glucose, and formiate. After intake of omega-3 EPUFA more pronounced differences were found in the choline/acetylcholine ratio. A significant increase in glucose and lactate, and a decrease in formiate and $\mathrm{N}$-acetylglucosamine were detected in the moderate DES compared to the mild DES patients ${ }^{31}$. A recent study has identified 156 metabolites in the tears of DES patients, among which 34 were significantly changed. The most prominent tear metabolites in DES patients were neutral lipids, cholesterol, lactate, glutamate, and glucose. On the other hand, lower levels of lactoferrin, lipocalin, and lipophilin indicate reduced antimicrobial defense and susceptibility to infections in DES patients. These results indicated that inflammatory related metabolites and pri- mary pathways like glycolysis and gluconeogenesis, amino acid metabolism may play regulatory role in DES (ref. ${ }^{44}$ ).

Non-targeted LC-MS/MS metabolomic study of serum metabolites in a British population sample (2819 participants) found an association between DES and decreased serum androgens. Significant decrease of androsterone sulfate and epiandrosterone sulfate and three other metabolites involved in androgen metabolism (4-androsten-3-beta, 17-beta-diol disulfate 1 and 2, and dehydroepiandrosterone sulfate) of all 222-screened metabolites were the most strongly associated with DES. Dryness and irritation symptoms were found to be strongly associated with decreased androgen metabolites. The authors consider that androgens might have a potential role in the development and treatment of DES (ref. ${ }^{45}$ ). Global and targeted metabonomic and proteomic analysis on metabolite profile of human conjunctival epithelial cells exposed to hyperosmotic stress revealed significant changes in 21 metabolites and 76 proteins in stressed cells vs. controls. Proteomic analysis indicated involvement of inflammatory pathways such as prostaglandin-2 synthesis and metabolism, interleukin-1, interleukin-10, interleukin-17 signaling pathways, and regulation of cytochrome $\mathrm{C}$ mediated apoptosis. Up regulation of keratins, plectin, tubulin-beta and vimentin in the stressed cells revealed the involvement of cytoskeleton remodeling. In addition, glycerophosphocholine synthesis and O-linked $\beta$-N-acetylglucosamine glycosylation were the key activated pathways in the stressed cells ${ }^{37}$.

\section{Keratoconus}

Keratoconus is a non-inflammatory disorder of the cornea characterized by progressive thinning, biomechanical weakening of the corneal stroma, cone-shaped protrusion of the cornea leading to irregular astigmatism and impairment in visual function. The onset of the condition is generally during puberty and typically progresses in the first three decades of life ${ }^{46}$.

The pathogenesis is still unclear, however, a variety of factors, including genetics and oxidative stress have been considered ${ }^{47}$. Using targeted LC-MS/MS metabolomic analysis a recent study detected more than 150 metabolites in human keratoconic cells most of which were related to activation of oxidative stress in keratoconus. The levels of lactate, lactate/malate and lactate/pyruvate ratios were elevated in human keratoconic cells, while arginine and reduced/oxidized glutathione ratio were reduced ${ }^{28,48}$. These results indicate that oxidative stress might play important role in keratoconus pathogenesis and progression.

Metabolic changes as a consequence of the disease can also be diagnosed from tears. Targeted LC-MS metabolomic analysis of tears in keratoconic patients without correction and keratoconic patients who wore rigid gaspermeable contact lenses vs. healthy subjects identified 296 different metabolites, more than 40 of which were significantly changed between the groups. The metabolites of the urea cycle, citric acid cycle, and oxidative stress were up regulated in keratoconic patients compared to healthy subjects ${ }^{28}$. 
Using the nano-LC/MS/MS approach, tears of 44 keratoconic patients were analyzed. Tear cytokeratins, matrix metalloproteinase 1 and 9 and mammoglobin B were increased in keratoconic patients, while lipocalin, lysozyme $\mathrm{C}$, immunoglobulin alpha and kappa and precursors to prolactin were deregulated ${ }^{49,50}$. In addition, decreased activity of lysyl oxidase and secreted-frizzled related protein 1 related to Wnt signaling pathway were demonstrated in the tears of 33 keratoconus patients ${ }^{51}$. Altogether these results indicate the activation of many pro-inflammatory factors and deregulation of oxidative stress in keratoconus. The synergistic effect of elevated inflammatory cytokines and matrix degrading enzymes finally leads to reduced collagens and collagen crosslinks typical for the disease. A specific term "Keratoconus Inflammaxis" was introduced describing the molecular fingerprint related to the disease ${ }^{49}$.

\section{Rhegmatogenous retinal detachment}

Retinal detachment is a term used to describe detachment of the neurosensory retina from the retinal pigment epithelium. Rhegmatogenous retinal detachment (RRD) is a potentially blinding condition and is the most common type of retinal detachment with an incidence reported between 6.3 and 17.9 per 100000 population. The pathogenesis of RRD depends on three factors retinal tear, vitreous liquefaction and traction. Retinal detachment occurs when the amount of the incoming fluid overwhelms the draining capacity of the retinal pigment epithelium. Once the posterior pole is involved, the photoreceptor cells of the retina will undergo apoptosis and degeneration. If not treated quickly, the rate of blindness is nearly $100 \%$. The pigment cells and fibroblasts will abnormally and ectopically proliferate - proliferative vitreoretinopathy (PVR), resulting, without treatment in irreversible impairment of the visual function ${ }^{52}$.

Untargeted metabolomic analysis using liquid chromatography-quadrupole-time-of-flight/mass spectrometry (LC-Q-TOF/MS) was used to analyze vitreous tissue of patients with RRD and RRD associated with choroidal detachment (RRDCD). The levels of 265 ions were significantly different in vitreous of patients with RRD and RRDCD. Twenty-four metabolites were identified and were mostly related to urea and tricarboxylic acid cycles. It is well known that the citric acid cycle plays a central role in providing energy for cell proliferation. Therefore, the changes of citric acid cycle between RRD and RRDCD groups might indicate a variation in proliferation. Succinate, lactate, and phenylpyruvate were higher in RRDCD indicating higher energy metabolism. The increase of uric acid in the RRDCD vs RRD group may be related to stimulation of vascular smooth muscle cell proliferation by the increased platelet-derived growth factor A-chain expression. Higher urea levels in RRDCD patients are associated with induced macrophage proliferation by inhibition the inducible nitric oxide synthase. The changes in lipid metabolites phytosphingosine, sphingosine, sphinganine and arachidonic acid levels may be related to inflammatory processes. Using the metabolomic approach, RRDCD and RRD were distinguished and the metabolites found to be different may be used as potential biomarkers for the diagnosis and treatment of the disease $^{53}$. Metabolomic analysis of vitreous samples from RRD patients with PVR, and control samples from donor eyes, identified 31 metabolites. Eleven of these were significantly different between RRD and PVR group. Most of the metabolites were associated with inflammation, proliferation and energy consumption. L-carnitine was significantly decreased in RRD than in PVR samples, which suggest that inflammation is more pronounced in RRD. On the other hand, in PVR samples ascorbate and valine levels were increased, while urea was decreased, suggesting more active fibroblast proliferation. The increase of citrate, succinate and D-glucuronolactone in RRD and PVR samples possibly reveals dysregulation of the citrate cycle $^{54}$.

\section{Proliferative vitreoretinopathy}

Proliferative vitreoretinopathy (PVR) is a sight-threatening complication of primary rhegmatogenous retinal detachment (RRD), severe diabetic retinopathy and intraocular trauma. It is defined as growth of membranes on both surfaces of the detached retina and on the posterior surface of the detached vitreous gel. PVR is not a distinct disease, but represents a complication common to a variety of clinical disorders. The incidence of PVR in primary retinal detachment ranges from 5.1 to $11.7 \%$ $\left(\right.$ ref. $\left.{ }^{55}\right)$. The molecular mechanisms of the disease remain unclear and have been the biggest problem for successful retinal reattachment. The pathogenesis includes proliferation and metaplasia of pigment epithelial cells and retinal glial cells. Contraction of the membranes results in retinal detachment, degeneration and visual loss. The prevention of PVR includes identification the patients at risk, using clinical (and perhaps biological) risk factors which may help for making the best decision for the PVR management ${ }^{56}$.

Metabolomic analysis based on reversed-phase liquid chromatography-quadrupol time-of-flight mass spectrometry identified 31 biomarkers in vitreous samples of PVR patients. The established set of biomarkers indicated disturbances in histidine metabolism and citrate cycle during PVR development. Pathway analysis revealed that the identified biomarkers are mainly related to disturbances in inflammation, proliferation, and energy consumption in the course of the disease $e^{48}$.

\section{Uveitis}

Uveitis is a term used to describe inflammatory processes of the portion of the eye known as uvea. It is a sight-threatening condition with diverse etiology that may lead to irreversible visual loss if not treated properly in a timely manner ${ }^{57}$. Uveitis is the fifth most common cause of severe visual loss in the developed world, and up to $20 \%$ of legal blindness is due to complications of uveitis. Based on its etiology, uveitis could be subdivided into infectious, non-infectious and masquerade. Lens-induced uveitis is a form of the disease which results from an immune response to lens proteins ${ }^{58}$. Symptoms and consequences can range from pain and redness to complete vision loss. 
Metabolomic analysis of vitreous humor samples was used to differentiate lens-induced and chronic uveitis. Using high resolution ${ }^{1} \mathrm{H}-\mathrm{NMR}$ spectroscopy and appropriate bioinformatics analysis a metabolite fingerprints in vitreous humor from patients with various vitreoretinal conditions were analyzed. A significant increase in oxaloacetate, glucose, and urea in lens-induced uveitis was indicated, while lactate, acetate and citrate levels were without changes in patients with chronic and lens-induced uveitis. According to Tan et al. ${ }^{48}$ these studies have not undergone validation required to identify these metabolites as disease biomarkers.

\section{Glaucoma}

Glaucoma is the second leading cause of blindness globally and the leading cause of irreversible visual loss. The term glaucoma represents a group of progressive optic neuropathies characterized by degeneration of retinal ganglion cells and as a result, changes in the optic nerve head ${ }^{59}$. Because of the slow course of the disease and lack of symptoms until late stage, diagnosis is frequently delayed. The pathogenesis of glaucoma remains unclear. The optic nerve damage is often caused by an abnormally high intra-ocular pressure determined by the balance between aqueous humor secretion and its drainage through the trabecular meshwork and uveoscleral outflow pathway. In patients with open-angle glaucoma (POAG), comprising nearly three-quarters of all glaucoma cases, there is increased resistance to aqueous outflow through the trabecular meshwork and as a result increased ocular pressure. In contrast, the drainage pathways are obstructed in patients with angle-closure glaucoma. Loss of ganglion cells, related to the level of intraocular pressure, leads to alterations in the visual field. Other potential damaged pathways are increased apoptosis (increased neurotoxicity, neurotrophin depletion) and oxidative stress ${ }^{60}$.

It has been shown that systemic conditions can also influence glaucoma pathogenesis. Therefore, metabolomic analysis of ocular and systemic samples will potentially contribute to a better understanding and early diagnosis of glaucoma. A recent case-control study performed non-targeted GC-TOF metabolomic analysis of aqueous humor samples of 40 POAG and 40 cataract patients served as controls. Potential metabolic biomarkers with areas under curve (AUC) from 0.62 to 0.86 for discrimination POAG patients from controls were 2-mercaptoethanesulfonic acid, D-erythronolactone, dehydroascorbic acid, galactose, mannose, pelargonic acid and ribitol. In addition, pathway analysis showed that biotin metabolism is highly impacted which could suggest that biotin plays a potentially important role in POAG pathophysiology $^{61}$. Flow injection analysis coupled with tandem mass spectrometry (FIA-MS/MS) and LC-MS/MS were used for analysis of 188 different endogenous molecules: free carnitine, acylcarnitines, hexoses, amino acids, biogenic amines, and lipids in plasma samples of 36 POAG patients and 27 age- and sex-matched controls. Significant changes in POAG patients compared to controls were found for 18 of the tested metabolites. The study indicated increased hexose concentration related to alterations in carbohydrate metabolism, higher levels of short- and medium-chain carnitines and a decrease in biogenic amines, spermine and spermidine. The accumulation of hexoses, short and medium acyl-carnitines as energy precursors assume potential mitochondrial dysfunction and energy metabolism changes. The decrease of spermine and spermidine may result in impaired regulation of mitochondrial membrane potential and deficient neuroprotection of the optic nerve. The authors conclude that spermine and spermidine might be key elements of POAG metabolic signature $^{62}$. A recent metabolomics study using non-targeted liquid chromatography coupled to a high resolution mass spectrometry (LC-HRMS) platform reported decreased levels of 9 metabolites (nicotinamide, hypoxanthine, xanthine, and 1-methyl-6,7-dihydroxy-1,2,3,4-tetrahydroisoquinoline) in the plasma samples of 34 POAG patients vs. 30 age- and sex-matched controls. It was concluded that nicotinamide deficiency is one of the most discriminating features. All these findings are in agreement with the hypothesis that mitochondrial impairment contributes to glaucoma pathogenesis. Another 5 metabolites ( $\mathrm{N}$-acetylL-leucine, arginine, RAC-glycerol 1-myristate, 1-oleoylRAC-glycerol, cystathionine) were found to be increased in POAG. The increase of arginine concentration may be related to altered arginine dependent nitric oxide regulatory pathway in glaucoma. These findings indicate deregulation of several metabolic pathways in glaucoma such as amino acid metabolism, energy supply, and nucleotide metabolism ${ }^{63}$. Metabolomic analysis of 26 aqueous humor samples of POAG patients and 26 age- and sex-matched non-POAG controls revealed statistically significant differences in 13 metabolites. The levels of taurine and spermine were reduced, while the concentrations of creatinine, carnitine, short-chain acylcarnitines, glutamine, glycine, alanine, leucine, isoleucine, hydroxyl-proline, and acetylornithine and, 7 phosphatidylcholines, one lysophosphatidylcholine, and one sphingomyelin were increased. The authors consider that alterations in taurine and creatinine may be related to impaired osmoprotection and thosee in spermine, taurine, and carnitine to deregulated neuroprotection. In addition, alterations in amino acid and phospholipid metabolism are important metabolic features of glaucoma ${ }^{64}$. A GC/TOF-MS metabolomic study of 50 aqueous humor samples from 30 patients with primary congenital glaucoma (PCG) indicated significant changes in metabolites associated with glycine, urea, and phenylalanine. In addition, genetic analysis revealed that the metabolites associated with these amino acids were linked with three glaucoma-associated single nucleotide polymorphisms (SNPs). Metabolic pathway analysis indicated that amino acid metabolism is mostly affected in PCG and probably plays an important role in the development of the disease. The authors consider that glycine could potentially serve as a noninvasive biomarker for PCG screening ${ }^{65}$. Targeted GC-MS metabolomic analysis of 38 serum samples of primary angle closure glaucoma (PACG) patients indicated significant changes in the long-chain unsaturated fatty acid profiles. Palmitoleic and $\gamma$-linolenic acid were significantly increased, while linoleic and arachidonic acid were reduced. Moreover, the levels 
of linoleic and arachidonic acid were negatively associated with intraocular pressure, while serum $\gamma$-linolenic acid levels were positively related to intraocular pressure. It might be concluded that long-chain unsaturated fatty acids palmitoleic, linoleic, $\gamma$-linolenic, and arachidonic acid are important metabolites associated with PACG and might have potential clinical significance for screening of the disease ${ }^{66}$.

\section{Age-related macular degeneration}

Age-related macular degeneration (AMD) is a major cause of blindness that affects more than $20 \%$ of the elderly population worldwide ${ }^{67}$. Early AMD is characterized by the presence of drusen and pigmentary abnormalities. In the late stage of AMD, central visual loss is a typical feature characterized by the presence of choroidal neovascularization and/or geographic atrophy. Accurate diagnosis includes clinical examination, like fundus photography, angiography, and optical coherence tomography ${ }^{67}$. At present, there is no cure for advanced AMD. Dietary antioxidant supplementation may slow the progression of the early forms. Supplement combination with vitamins C and $\mathrm{E}$, beta-carotene, and zinc leads to a delay in progression of the disease in patients with intermediate AMD.

Many risk factors contribute to development of any AMD including age and genetics. In addition, environmental factors such as sunlight, diet (high fat intake), lifestyle (smoking and alcohol consumption) can also contribute to the risk of AMD (ref. ${ }^{68}$ ). More than 50 genetic variants are associated with the disease and are mainly related to changes in serum cholesterol and triglyceride levels ${ }^{69}$. In addition, the exchange of metabolites between the retina, retinal pigment epithelium, and choroid complex provide a basis for local and systemic metabolomic analysis in AMD for finding relevant biomarkers that are indicative of AMD development or progression prior to clinical manifestation.

A recent cross-sectional metabolomics study using nontargeted ultra-high performance liquid chromatography-tandem MS (UPLC-MS/MS) on 391 AMD patients and 100 controls revealed significant difference in 28 plasma metabolites between diseased patients and controls, most of which also differed across AMD severity stages. The most prominent differences were in lipid profile, followed by amino acid and nucleotide profile. Pathway analysis indicated significant changes in glycerophospholipid, purine, taurine and hypotaurine, and nitrogen metabolism not only between the diseased and control group, but also across severity stages of AMD. The authors consider that alterations in glycerophospholipids may be related to activation of oxidative stress in AMD. The dysregulation of purine, taurine and hypotaurine metabolism may be related to deregulation of cellular energy metabolism, disturbed antioxidant defense and neuroprotection. Glutamate and glutamine changes between AMD patients and controls as well as across AMD severity stages are possibly associated with disturbances in neurotransmitter supply, important for retina and visual pathway ${ }^{70,71}$. GCMS/MS study on plasma metabolome profile of AMD patients and controls also reported disturbed amino acid metabolism in AMD patients. Significant changes were found in N-acetyl-L-alanine, L-tyrosine, L-phenylalanine, L-methionine, and L-arginine, which leads to the conclusion that AMD may be also related to disorders in amino acid metabolism ${ }^{72}$. ${ }^{1} \mathrm{H}$ NMR spectroscopy study on urinary metabolomic signatures of patients with different stages of AMD and a control group reported depletion of some amino acids and citrate in adult AMD patients vs controls $^{71}$. Serum GC-TOF-MS metabolome study on 446 patients with macular neovascularization (AMD, polypoidal choroidal vasculopathy, PCV, and pathological myopia, PM) and 138 cataract adults as controls, identified significant differences in 33 metabolites in the patients with macular neovascularization. The phenotypic distribution of metabolic pathway alterations revealed that mostly affected in AMD were the pentose phosphate pathway and mitochondrial electron transport chain, in PCV purine metabolism and glycolysis, and in PM thiamine and purine metabolism ${ }^{73}$.

Metabolomic study using HPLC-QTOF-MS platform on the metabolic changes related to photoreceptor degeneration in rat retinal tissue indicated 203 significantly changed metabolites in the diseased group. Most of these were phospholipids, including glycerophosphocholines, glycerophosphoethanolamines, long-chain acylcarnitines, monoacylglycerols, fatty acid amides, long-chain polyunsaturated fatty acids (LC-PUFAs) and various oxidized species. Decreased levels of acylcarnitines, docosahexanoic acid, all-trans retinal were indicated in the dystrophic retinal tissue, assumed disturbed retinal homeostasis and dysregulated visual cycle ${ }^{74}$. Targeted metabolomic analysis revealed decreased risk of neovascular AMD progression in patients with higher plasma omega-3 LC-PUFAs such as EPA and DHA (ref. ${ }^{75}$ ). In a recent review, metabolites related to oxidative stress, complement system, and lipid metabolism were discussed as potential biomarker-candidates for AMD $\left(\right.$ ref. $\left.^{76}\right)$.

\section{Diabetic retinopathy}

Diabetic retinopathy (DR) is the most common complication of diabetes mellitus and a major cause of vision loss in middle-aged and elderly people ${ }^{77}$. DR is divided into two forms: non-proliferative diabetic retinopathy (NPDR) and proliferative diabetic retinopathy (PDR). NPDR is characterized by micro aneurysms, retinal hemorrhages, intraretinal microvascular abnormalities, and venous caliber changes, while the hallmark feature of PDR is pathologic pre-retinal neovascularization ${ }^{78}$. The most common cause of vision loss in patients with DR is diabetic macular edema. The treatment of DR remains challenging and includes anti-vascular endothelial growth factor (VEGF) agents, laser treatment, steroids and surgical interventions.

One of the first metabolomics studies on plasma samples of type 2 diabetes mellitus patients with moderate non proliferative DR using GC-MS platform revealed significantly altered levels of 11 metabolites ${ }^{79}$. Decreased levels of 1,5-anhydroglucitol and increased 
levels of 1,5-gluconolactone, 2-deoxyribonic acid, 3,4-dihydroxybutyric acid, erythritol, gluconic acid, lactose/ cellobiose, maltose/trehalose, mannose, ribose, and urea were reported. In the validation set, 2-deoxyribonic acid, 3,4-dihydroxybutyric acid, erythritol, gluconic acid, and ribose were found to remain elevated, while maltose was decreased. Increased concentrations of cytosine, cytidine, and thymidine were also found to be associated with DR compared to the control subjects. Of these compounds, the highest area under the curve, sensitivity (73.7\%) and specificity $(91.9 \%)$ was found for cytidine, suggesting it could be a potential biomarker for the disease.

Global and targeted LC-MS metabolomic analysis of vitreous samples from 20 patients with type 2 diabetes and proliferative DR and 31 controls with no diabetes revealed dysregulation of methionine, allantoin, decanoylcarnitine, arginine, proline, citrulline, ornithine, and octanoylcarnitine in patients with PDR (ref. ${ }^{80}$ ). In the retina, arginine is metabolized by the arginase pathway to ornithine and urea and by nitric oxide synthase to citrulline and nitric oxide (NO). It is suggested that hyperactivity of the arginase pathway may result in reduced NO availability, impaired vasodilation, endothelial dysfunction, increased generation of oxygen and nitrogen reactive species, characteristic for DR (ref. ${ }^{81}$ ).

Recently ${ }^{1} \mathrm{H}$-NMR-based metabolomic study on aqueous humor samples of patients with diabetes mellitus and cataract, DR and cataract, and senile cataract using 2-dimensional pulsed-field gradient correlation spectroscopy (gCOSY) and 2D homonuclear total correlation spectroscopy (TOCSY) identified 25 principal metabolites. Of these, the levels of lactate and succinate were dramatically decreased, while asparagine, glutamine, histidine, and threonine were increased in patients with DR compared to diabetes patients without cataract. Data analysis revealed that lactate, succinate, 2-hydroxybutyrate, asparagine, dimethylamine, histidine, threonine, and glutamine were the most altered metabolites that potentially play roles in the development and progression of DR. Metabolic pathway analysis showed that over 11 pathways were influenced including alanine, aspartate and glutamate metabolism, aminoacyl-tRNA biosynthesis, propanoate, and nitrogen metabolism $^{82}$. The detected alterations in amino acid metabolism are possibly related to mitochondrial dysfunction, oxidative stress and endothelial damage in patients with DR.

\section{CONCLUSION}

Metabolomics is a powerful tool for studying the pathogenesis of a disease. The identification of new biomarkers for disease development and progression, which can be integrated into the currently available clinical diagnostic and prognostic detection tools, is one of the most promising applications of metabolomics in medicine. In addition, metabolomic analysis and profiling can provide further knowledge regarding the pathophysiology of ocular diseases and opportunities for clinical intervention at early stages of the disease as well as for new drug development. Ideally, in the future, all treatments should be performed under metabolomic control.

\section{Search strategy and selection criteria}

Scientific articles on metabolomic analysis for identification of biomarkers in ocular diseases published in the last five years (2015-2020) were searched using the PubMed and Web of Science databases. The search approach involved the key words "biomarkers", "metabolomic analysis", "mass spectroscopy," "nuclear magnetic resonance", "ocular diseases", "macular degeneration”, "dry eye", "keratoconus", "retinal detachment", "inflammatory eye disease," "glaucoma", and "diabetic retinopathy”. Only studies published in English were reviewed.

Author contributions: NN-T: literature search concerning the bioanalytical part of metabolomic analysis and the biomarkers study; draft manuscript writing; MR: literature search concerning the clinical aspect of the ocular diseases discussed in the manuscript; draft manuscript writing; BG: final writing the manuscript, editing according to journal requirements; $\mathrm{CG}$ : final corrections and critical reading of the manuscript.

Conflict of interest statement: The authors state that there are no conflicts of interest regarding the publication of this article.

\section{REFERENCES}

1. Nalbantoglu S. Metabolomics: Basic Principles and Strategies. Molecular Medicine, Sinem Nalbantoglu and Hakima Amri, IntechOpen [on the internet] 2019 Aug [cited 2019 Aug 7]; Available from: https://www.intechopen.com/books/molecular-medicine/ metabolomics-basic-principles-and-strategies. doi: 10.5772/intechopen.88563

2. PubMed US National Library of Medicine National Institutes of Health. Available from: https://www.ncbi.nlm.nih.gov/pubmed

3. METLIN. Available from: https://metlin.scripps.edu/landing_page. php?pgcontent=mainPage

4. Lindon JC, Nicholson JK, Holmes E, editors. The Handbook of Metabolic Phenotyping. 1st ed. Amsterdam: Elsevier; 2019.

5. Nicholson JK, Darzi AV, Holmes E, Lindon JC, editors. Metabolic Phenotyping in Personalized and Public Healthcare. 1st ed. New York: Academic Press; 2016.

6. Noreldeen HAA, Liu X, Xu G. Metabolomics of lung cancer: Analytical platforms and their applications. J Sep Sci 2020;43(1):120-33.

7. Vinaixa M, Schymanski EL, Neumann S, Navarro M, Sale R, Yanes Ó. Mass spectral databases for LC/MS- and GC/MS-based metabolomics: State of the field and future prospects. Trends Analyt Chem 2016;78,23-35.

8 Chen W, Li S, Kulkarni AS, Huang L, Cao J, Qian K, Wan J. Single Cell Omics: From Assay Design to Biomedical Application. Biotechnol J [serial on the Internet]. 2019 Oct [cited 2019 Oct 21]; 15(1):[about 10 p.]. Available from: https://onlinelibrary.wiley.com/doi/ epdf/10.1002/biot.201900262.

9. Alonso A, Marsal S, Julià A. Analytical methods in untargeted metabolomics: state of the art in 2015. Front Bioeng Biotechnol [serial on the Internet]. 2015 Mar [cited 2015 Mar 5]; 3:[about 20 p. Available from: https://www.ncbi.nlm.nih.gov/pmc/articles/PMC4350445/

10. Gika H, Virgiliou C, Theodoridis G, Plumb RS, Wilson ID. Untargeted LC/MS-based metabolic phenotyping (metabonomics/metabolomics): The state of the art. J Chromatogr B Analyt Technol Biomed Life Sci 2019;1(1117):136-47.

11. Schrimpe-Rutledge AC, Codreanu SG, Sherrod SD, McLean JA. 
Untargeted Metabolomics Strategies-Challenges and Emerging Directions. J Am Soc Mass Spectrom 2016;27(12):1897-05.

12. Silva RA, Pereira TCS, Souza AR, Ribeiro PR. 1 H NMR-based metabolite profiling for biomarker identification. Clin Chim Acta 2020;502:26979.

13. Emwas A-H, Roy R, McKay RT, Tenori L, Saccenti E, Gowda GAN, Raftery D, Alahmari F, Jaremko L, Jaremko M, Wishart DS Spectroscopy for Metabolomics Research. Metabolites [serial on the internet]. 2019 Jun [cited 2019 Jun 27];9(7):[about 39 p.]. Available from: https://www.ncbi.nlm.nih.gov/pmc/articles/PMC6680826/

14. Clendinen CS., Pasquel C, Ajredini R, Edison AS. 13C NMR Metabolomics: INADEQUATE Network Analysis. Anal Chem 2015;87(11):5698-5706.

15. Alsaleh M, Barbera TA, Andrews RH, Sithithaworn P, Khuntikeo N Loilome W, Yongvanit P, Cox IJ, Syms RRA, Holmes E, Taylor-Robinson SD. Mass Spectrometry: A Guide for the Clinician. J Clin Exp Hepatol 2019;9(5):597-06.

16. Zhou J, Liu H, Liu Y, Liu J, Zhao X., Yin Y. Development and Evaluation of a Parallel eaction Monitoring Strategy for Large-Scale Targeted Metabolomics Quantification. Analytical Chemistry 2016;88(8):447886.

17. Use of Metabolomics to Advance Research on Environmental Exposures and the Human Exposome. In: Workshop in Brief. National Academies of Sciences, Engineering, and Medicine, Division on Earth and Life Studies; Board on Life Sciences. Washington, DC, USA. The National Academies Press; 2016

18. Vernocchi P, Del Chierico F, Putignani L. Gut Microbiota Profiling: Metabolomics Based Approach to Unravel Compounds Affecting Human Health. Front Microbiol [serial on the Internet]. $2016 \mathrm{Jul}$ [cited 2016 Jul 26];7:[about 21 p.]. Available from: https://www.ncbi. nlm.nih.gov/pmc/articles/PMC4960240/

19. van der Hooft JJ, Wandy J, Young F, Padmanabhan S, Gerasimidis K, Burgess KEV, Barrett MP, Rogers S. Unsupervised Discovery and Comparison of Structural Families Across Multiple Samples in Untargeted Metabolomics. Anal Chem 2017;89(14):7569-77.

20. Heinemann J. Cluster Analysis of Untargeted Metabolomic. Experiments. Methods Mol Biol 2017;1859:275-85.

21. Blasco H, Błaszczynski, Billau JC H, Nadal-Desbarats L, Pradat PF, Devos D, Moreau C, Andres CR, Emond P, Corcia P, Słowiński R Comparative analysis of targeted metabolomics: Dominance-based rough set approach versus orthogonal partial least square-discriminant analysis. J Biomedi Informa 2015;53:291-9.

22. Everett JR, Holmes E, Veselkov KA, Lindon JC, Nicholson JK. A Unified Conceptual Framework for Metabolic Phenotyping in Diagnosis and Prognosis. Trends Pharmacol Sci 2019;40(10):763-73.

23. Burgess LG, Uppal K, Walker DI Roberson RM, Tran V, Parks MB, Wade EA, May AT, Umfress AC, Jarrell KL, Stanley BOC, Kuchtey J, Kuchtey RW, Jones DP, Brantley MA Jr. Metabolome wide association study of primary open angle glaucoma. Invest Ophthalmol Vis Sci 2015;56:5020-8.

24. Sidek S, Ramli N, Rahmat K, Ramli NM, Abdulrahman F, Kuo TL. In vivo proton magnetic resonance spectroscopy (1H-MRS) evaluation of the metabolite concentration of optic radiation in primary open angle glaucoma. Eur Radiol 2016;26:4404-12.

25. Laíns I, Chung W, Kelly RS Gil J, Marques M, Barreto P, Murta JN, Kim IK, Vavvas DG, Miller JB, Silva R, Lasky-Su J, Liang L, Miller JW, Husain D. Human Plasma Metabolomics in Age-Related Macular Degeneration: Meta-Analysis of Two Cohorts. Metabolites 2019;9(7):127. doi: 10.3390/metabo9070127

26. Laíns I, Duarte D, Barros AS Martins, AS, Gil J, Miller JB, Marques M, Mesquita T, Kim IK, Cachulo ML, Vavvas D, Carreira IM, Murta JN, Silva R, Miller JW, Husain D, Gil AM. Human plasma metabolomics in age-related macular degeneration (AMD) using nuclear magnetic resonance spectroscopy. PLoS One [serial on the Internet] 2017 May [cited 2017 May 18]; 12(5):[about 18 p.]. Available from: https://europepmc.org/backend/ptpmcrender.fcgi?accid=PMC5436712\&blo btype $=$ pdf

27. Huiyi J, Bijun Z, Xia L, Jing J, Haidong Z. Metabolic characterization of diabetic retinopathy: An 1H-NMR-based metabolomic approach using human aqueous humor. J Pharm Biomed Anal 2019;174:41421.

28. Karamichos D, Zieske JD, Sejersen H, Sarker-Nag A, Asara JM, Hjortdal J. Tear metabolite changes in keratoconus. Exp Eye Res 2015;132:1-8,

29. Yu M, Wu Z, Zhang Z, Huang X, Zhang Q. Metabolomic Analysis of Human Vitreous in Rhegmatogenous Retinal Detachment Associated With Choroidal Detachment. Invest Ophthalmol Vis Sci 2015;56(9):5706-13.

30. Wang H, Zhai R, Sun Q, Wu Y, Wang Z, Fang J, Kong X. Metabolomic Profile of Posner-Schlossman Syndrome: A Gas Chromatography Time-of-Flight Mass Spectrometry-Based Approach Using Aqueous Humor. Front Pharmacol [serial on the Internet]. 2019 Nov [cited 2019 Nov 7]; 10:[about 11 p.]. Available from: https://www.ncbi.nlm. nih.gov/pmc/articles/PMC6855217/

31. Galbis-Estrada C, Pinazo-Duran MD, Martinez-Castillo S, Morales JM, Monleon D, Zanon-Moreno V. A metabolomic approach to dry eye disorders: the role of oral supplements with antioxidants and omega 3 fatty acids. Mol Vis 2015;21:555-67.

32. Munoz-Hernandez AM, Galbis-Estrada C, Santos-Bueso E, CuiñaSardiña R, Díaz-Valle D, Gegúndez-Fernández JA, Pinazo-Durán MD, Benítez-del-Castillo JM. Human tear metabolome. Arch Soc Esp Oftalmol 2016;91:157-9.

33. Barbas-Bernardos C, Armitage EG, Garcia A, Mérida S, Navea A, Bosch-Morell F, Barbas C. Looking into aqueous humor through metabolomics spectacles - exploring its metabolic characteristics in relation to myopia. J Pharm Biomed Anal 2016;127:18-25.

34. Ji Y, Rao J, Rong X, Lou S, Zheng Z, Lu Y. Metabolic characterization of human aqueous humor in relation to high myopia. Exp Eye Res 2017;159:147-55.

35. Patnaik S, Jalali S, Joshi MB, Satyamoorthy K, Kaur I. Metabolomics Applicable to Retinal Vascular Diseases. Methods Mol Biol 2019;1996:325-31.

36. Priyadarsini S, McKay TB, Sarker-Nag A, Allegood J, Chalfant C, Ma $J-X$, Karamichos D. Complete metabolome and lipidome analysis reveals novel biomarkers in the human diabetic corneal stroma. Exp. Eye Res 2016;153:90-100.

37. Chen L, Li J, Guo T, Ghosh S, Ghosh S, Koh SK, Tian D, Zhang L, Jia D, Beuerman RW, Aebersold R, Chan ECY, Zhou L. Global metabonomic and proteomic analysis of human conjunctival epithelial cells (IOBA-NHC) in response to hyperosmotic stress. J Proteome Res 2015;14:3982-95.

38. Tsentalovich YP, Verkhovod TD, Yanshole VV, Kiryutin AS, Yanshole LV, Fursova AZ, Stepakov DA, Novoselov VP, Sagdeev RZ. Metabolomic composition of normal aged and cataractous human lenses. Exp. Eye Res 2015;134:15-23.

39. Craig JP, Nichols KK, Nichols JJ, Caffery B, Dua HS, Joo CK, Liu Z, Nelson JD, Nichols JJ, Tsubota K, Stapleton F. FOS DEWS II Definition and Classification Report. Ocular Surface 2017;15(3):276-83.

40. Kanellopoulos, AJ, Asimellis G. In pursuit of objective dry eye screening clinical techniques. Eye Vis (Lond) [serial on the Internet]. 2016 Jan [cited 2016 Jan 18];3:[ about 7 p.]. Available from: https://www. ncbi.nlm.nih.gov/pmc/articles/PMC4716631/

41. Aggarwal S, Galor A. What's new in dry eye disease diagnosis? Current advances and challenges. F1000Res [serial on the Internet]. 2018 Dec [cited 2018 Jun 19];7:[about 7 p.]. Available from: https:// www.ncbi.nlm.nih.gov/pmc/articles/PMC6305205/

42. Zhang X, Jeyalatha VM, Yangluowa Q, Qu Y, He X, Ou S, Bu J, Jia C, Wang J, Wu H, Liu Z, Li W. Dry eye management: Targeting the ocular surface microenvironment. Int J Mol Sci [serial on the Internet]. 2017 Jun [cited 2017 Jun 29]; 18(7):[about 28 p.]. 1398. Available from: https://www.ncbi.nlm.nih.gov/pmc/articles/PMC5535891/

43. Yazdani $M$, Benedikte $K$, Elgstøen $P$, Rootwelt $H$, Shahdadfar $A$, Utheim $\varnothing A$, Utheim TP. Tear Metabolomics in Dry Eye Disease: A Review. Int J Mol Sci [serial on the Internet]. 2019 Aug [cited 2019 Aug 1]; 20(15):[about 18 p.]. Available from: https://www.ncbi.nlm. nih.gov/pmc/articles/PMC6695908/

44. Chen X, Rao J, Zheng Z, Yu Y, Lou S, Liu L, He Q, Wu L, Sun X. Integrated Tear Proteome and Metabolome Reveal Panels of Inflammatory-Related Molecules via Key Regulatory Pathways in Dry Eye Syndrome. J. Proteome Res 2019;18(5):2321-30.

45. Vehof J, Hysi PG, Hammond. CJ. A Metabolome-Wide Study of Dry Eye Disease Reveals Serum Androgens as Biomarkers. Ophthalmology 2017;124(4):505-11.

46. Millodot M, Ortenberg I, Lahav-Yacouel K, Behrman S. Effect of ageing on keratoconic corneas. J Optom 2016;9(2),72-7.

47. Soiberman U, Foster JW, Jun AS, Chakravarti S.Pathophysiology of Keratoconus: What Do We Know Today. Open Ophthalmol J 2017;11:252-61.

48. Tan SZ, Begley P, Mullard G, Hollywood KA, Bishop PN. Introduction 
to metabolomics and its applications in ophthalmology. Eye 2016;30:773-83

49. Nishtala K, Pahuja N, Shetty R, Rudy MM, Nuijts A, Ghosh A. Tear biomarkers for keratoconus. Eye Vis (Lond) [serial on the Internet]. 2016 Aug [cited 2016 Aug 4]; 3:[about 7 p.]. Available from: https:// www.ncbi.nlm.nih.gov/pmc/articles/PMC4973115/

50. Shetty R, Ghosh A, Lim RR, Subramani M, Mihir K, Reshma AR Ranganath A, Nagaraj S, Nuijts RMM, Beuerman R, Shetty R, Das D Chaurasia SS, Sinha-Roy A, Ghosh A. Elevated Expression of Matrix Metalloproteinase-9 and Inflammatory Cytokines in Keratoconus Patients Is Inhibited by Cyclosporine A. Invest Ophthalmol Vis Sci 2015;56(2):738-50.

51. You J, Hodge C, Wen L, McAvoy JW, Madigan MC, Sutton G. Tear levels of SFRP1 are significantly reduced in keratoconus patients. Molecular Vision 2013;19:509-15.

52. Liao L, Zhu X-H. Advances in the treatment of rhegmatogenous retinal detachment. Int J Ophthalmol 2019;12:660-7.

53. Yu M, Wu Z, Zhang Z, Huang X, Zhang Q. Metabolomic analysis of human vitreous in rhegmatogenous retinal detachment associated with choroidal detachment. Invest Ophthalmol Vis Sci 2015;56:570613.

54. Laínsa I, Gantnerc M, Murinelloc S, Lasky-Su JA, Miller JW, Friedlander M5, Husain D. Metabolomics in the study of retinal health and disease. Prog Retin Eye Res 2019;69:57-79.

55. Kwon OW, Song JH, Roh MI. Retinal detachment and proliferative vitreoretinopathy. Dev Ophthalmol 2015;55:154-62.

56. Ciprian D. The Pathogeny of Proliferative Vitreoretinopathy. Rom J Ophthalmol 2015;59(2):88-92.

57. Chen SC, Sheu SJ. Recent advances in managing and understanding uveitis. F1000 Research F1000Res [serial on the Internet]. 2017 Mar [cited 2017 Mar 16];6:[about 9 p.]. Available from: https://www.ncbi. nlm.nih.gov/pmc/articles/PMC5357029/

58. Kanski J Bowling B. Kanski's Clinical Ophthalmology. A Systematic Approach. New York: Elsevier; 2016.

59. Jonas JB, Aung T, Bourne RR, Bron AM, Ritch R, Panda-Jonas S Glaucoma. Lancet 2017;390(10108):2183-93.

60. Barbosa-Breda J, Himmelreich U, Ghesquière B, Rocha-Sousa A Stalmans I. Clinical Metabolomics and Glaucoma. Ophthalmic Res 2018;59;1-6.

61. Pan C, Ke C Chen Q, Tao Y, Zha X, Zhang YP, Zhong H. Differential metabolic markers associated with primary open-angle glaucoma and cataract in human aqueous humor. BMC Ophthalmol [serial on the Internet]. 2020 May [cited 2020 May 6]; 20: [about 8 p.]. Available from: https://www.ncbi.nlm.nih.gov/pmc/articles/PMC7203853/ pdf/12886_2020_Article_1452.pdf

62. Leruez S, Marill A, Bresson T, de Saint Martin G, Buisset A, Muller J, Tessier L, Gadras C, Verny C, Gohier P, Amati-Bonneau P, Lenaers G, Bonneau D, Simard G, Milea D, Procaccio V, Reynier P, de la Barca JMC. A Metabolomics Profiling of Glaucoma Points to Mitochondrial Dysfunction, Senescence, and Polyamines Deficiency. Invest Ophthalmol Vis Sci 2018;59(11):4355-61.

63. Nzoughet JK, Guehlouz K, Leruez S, Gohier P, Bocca C, Muller J, Blanchet O, Bonneau D, Simard G, Milea D, Procaccio V, Lenaers G, de la Barca JMC, Reynier P. A Data Mining Metabolomics Exploration of Glaucoma. Metabolites [serial on the Internet] 2020 Jan [cited 2020 Jan 28];10(2):[about 14 p.]. Available from: https://www.ncbi. nlm.nih.gov/pmc/articles/PMC7074047/

64. Buisset A, Gohier P, Leruez S, Muller J, Amati-Bonneau P, Lenaers G, Bonneau D, Simard G, Procaccio V, Annweiler C, Milea D, Reynier P, de la Barca JMC. Metabolomic Profiling of Aqueous Humor in Glaucoma Points to Taurine and Spermine Deficiency: Findings from the Eye-D Study. J Proteome Res 2019;18(3):1307-15.

65. Chen X, Chen Y, Wang L, Sun X. Metabolomics of the aqueous humor in patients with primary congenital glaucoma. Mol Vis 2019;25:48901.

66. Rong S, Li Y, Guan Y, Zhu L, Zhou Q, Gao M, Pan H, Zou L, Chang D. Long-chain unsaturated fatty acids as possible important metabolites for primary angle-closure glaucoma based on targeted metabolomic analysis. Biomed Chromatogr [serial on the Internet] 2017 Feb [cited Feb 18]; 31(9):[about 20 p.]. Available from: https:// onlinelibrary.wiley.com/doi/epdf/10.1002/bmc.3963

67. Mitchell P, Liew G, Gopinath B, Wong TY. Age-related macular degeneration. Lancet 2018;392(10153):1147-59.
68. Armstrong RA, Mousavi M. Overview of Risk Factors for Age-Related Macular Degeneration (AMD). J Stem Cells 2015;10(3):171-91.

69. Fritsche LG, Igl W, Bailey JN, Grassmann F, Sengupta S, BraggGresham JL, Burdon KP Hebbring SJ, Wen C, Gorski M, Kim IK, Cho D, Zack D, Souied E, Scholl HPN, Bala E, Lee KEHunter, DJ, Sardell RJ, Mitchell P, Merriam JE, Cipriani V, Hoffman JD, Schick T, Lechanteur YTE, Guymer RH, Johnson MP, Jiang Y, Stanton CM, Buitendijk GHS, Zhan X, Kwong AM, Boleda A, Brooks M, Gieser L, Ratnapriya R, Branham KE, Foerster JR, Heckenlively JR, Othman MI, Vote BJ, Liang HH, Souzeau E, McAllister IL, Isaacs T, Hall J, Lake S, Mackey DA, Constable IJ, Craig JE, Kitchner TE, Yang Z, Su Z, Luo H, Chen D, Ouyang $H$, Flagg K, Lin D, Mao G, Ferreyra H, Stark K, von Strachwitz CN, Wolf A, Brandl C, Rudolph G, Olden M, Morrison MA, Morgan DJ, Schu M Ahn J, Silvestri G, Tsironi EE, Park KH, Farrer LA, Orlin A, Brucker A, Li M, Curcio C, Mohand-Saïd S, Sahel J-A, Audo I, Benchaboune M, Cree AJ, Rennie CA, Goverdhan SV, Grunin M, Hagbi-Levi S, Campochiaro P, Katsanis N, Holz FG, Blond F, Blanché $H$, Deleuze JF,. Igo RP, Jr., Truitt B, Peachey NS, Meuer SM, Myers CE, Moore EL, Klein R, Hauser MA, Postel EA, Courtenay MD, Schwartz SG, Kovach JL, Scott WK, Liew G,. Tfan AG, Gopinath B, Merriam JC, Smith RT, Khan JC, Shahid H, Moore AT, McGrath JA, Laux R, Brantley MA, Jr., Agarwal A, Ersoy L, Caramoy A, Langmann T, Saksens NTM, de Jong EK, Hoyng CB, Cain MS, Richardson AJ, Martin TM, Blangero J, Weeks DE, Dhillon B, van Duijn CM, Doheny KF, Romm J, Klaver CC, Hayward, C, Gorin MB, Klein ML, Baird PN, den Hollander AI Fauser S, Yates JRW, Allikmets R, Wang JJ, Schaumberg DA, Klein BEK, Hagstrom SA, Chowers I, Lotery AJ, Léveillard T, Zhang K, Brilliant MH, Hewitt AW, Swaroop A, Chew EY, Pericak-Vance MA, DeAngelis M, Stambolian D, Haines JL, lyengar SK, Weber BHF, Abecasis GR, Heid IM. A large genome-wide association study of age-related macular degeneration highlights contributions of rare and common variants. Nat Genet 2016;48(2):134-43.

70. Laíns I, Kelly RS, Miller JB, Silva R, Vavvas DG, Kim IK, Murta JN, LaskySu J, Miller JW, Husain D. Human Plasma Metabolomics Study across All Stages of Age-Related Macular Degeneration Identifies Potential Lipid Biomarkers. Ophthalmology 2018;125(2):245-54.

71. Laíns I, Duarte D, Barros AS, Martins AS, Carneiro TJ, Gil JQ, Miller JB, Marques M, Mesquita TS, Barreto P, Kim IK da Luz Cachulo M, Vavvas DG, Carreira IM, Murta JN, Silva R, Miller JW, Husain D, Gil AM. Urine Nuclear Magnetic Resonance (NMR) Metabolomics in Age-Related Macular Degeneration. J Proteome Res 2019;18(3):1278-88.

72. Luo D, Deng T, Yuan W, Deng H, Jin M. Plasma metabolomic study in Chinese patients with wet age-related macular degeneration. BMC Ophthalmol [serial on the Internet]. 2017 Sep [cited 2017 Sep 6];17(1):[about 9 p.]. Available from: https://www.ncbi.nlm.nih.gov/ pmc/articles/PMC5585971/

73. Liu K, Fang J, Jin J. Serum Metabolomics Reveals Personalized Metabolic Patterns for Macular Neovascular Disease Patient Stratification. J Proteome Res 2020;19(2):699-07.

74. Brown CN, Green BD, Thompson RB, den Hollander Al, Lengyel I, and on behalf of the EYE-RISK consortium. Metabolomics and AgeRelated Macular Degeneration. Metabolites 2019;9(4):1-36.

75. Wang J, Westenskow PD, Fang M, Friedlander M, Siuzdak G. Quantitative metabolomics of photoreceptor degeneration and the effects of stem cell-derived retinal pigment epithelium transplantation. Philos Trans A Math Phys Eng Sci [serial on the Internet]. 2016 Oct [cited 2016 Oct 28]; 374(2079):[about 15 p.]. Available from: https://www.ncbi.nlm.nih.gov/pmc/articles/PMC5031641/

76. Kersten E, Paun CC, Schellevis RL, Hoyng CB, Delcourt C, Lengyel I, Peto T, Ueffing M, Klaver CCW, Dammeier S, I den Hollander A, de Jong EK. Systemic and ocular fluid compounds as potential biomarkers in age-related macular degeneration. Surv Ophthalmol 2018:63:9-39.

77. Wong TY, Cheung CM, Larsen M, Sharma S, Simó R. Diabetic retinopathy. Nat Rev Dis Primers. [serial on the Internet] 2016 Mar [cited 2016 Mar 17]; 2:[about 12 p.]. Available from: https://www.nature. com/articles/nrdp201612

78. Wang W, Lo ACY. Diabetic Retinopathy: Pathophysiology and Treatments. Int J Mol Sci [serial on the Internet]. 2018 Jun [cited 2018 Jun 20];19(6):[about 14 p.]. Available from: https://www.ncbi. nlm.nih.gov/pmc/articles/PMC6032159/

79. Chen L, Cheng CY, Choi H, Ikram MK, Sabanayagam C, Tan GSW, Tian D, Zhang L, Venkatesan G, Tai ES, Wang JJ, Mitchell P, Cheung CMG, 
Beuerman RW, Zhou L, Chan ECY, Wong TY. Plasma Metabonomic Profiling of Diabetic Retinopathy. Diabetes 2016;65(4):1099-108.

80. Paris LP, Johnson $C H$, Aguilar E, Usui Y, Cho K Hoang LT, Feitelberg $D$, Benton HP, Westenskow PD, Kurihara T, Trombley J, Tsubota K, Ueda S, Wakabayashi Y, Patti GJ, Ivanisevic J, Siuzdak G, Friedlander M Global metabolomics reveals metabolic dysregulation in ischemic retinopathy. Metabolomics [serial on the Internet]. 2015 Nov [cited 2015 Nov 18];12:[about 10 p.]. Available from: https://www.ncbi.nlm. nih.gov/pmc/articles/PMC4651979/
81. Liew G, Lei Z, Tan G, Joachim N, Ho I, Wong TY, Mitchell P, Gopinath B, Crossett B. Metabolomics of Diabetic Retinopathy. Curr Diab Rep [serial on the Internet]. 2017 Sep [cited 2017 Sep 23];17(11):[about 15 p.]. 102. Available from: https://pubmed.ncbi.nlm.nih. gov/28940103/

82. Jin H, Zhu B, Liu X, Jin J, Zou H. Metabolic characterization of diabetic retinopathy: An $1 \mathrm{H}-\mathrm{NMR}$-based metabolomic approach using human aqueous humor. J Pharm Biomed Anal 2019;174:414-21. 